\title{
Comparisons of statistical multifragmentation and evaporation models for heavy-ion collisions
}

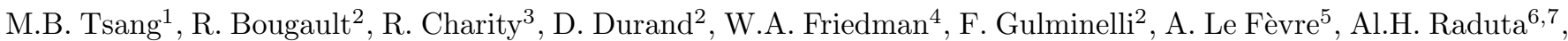

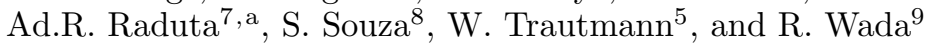 \\ 1 National Superconducting Cyclotron Laboratory and Department of Physics and Astronomy, Michigan State University, East \\ Lansing, MI 48824, USA \\ ${ }^{2}$ LPC/Ensicaen and University of Caen, 6 Bd du Maréchal Juin, F-14050 Caen cedex, France \\ 3 Chemistry Department, Washington University, St. Louis, MO 63130, USA \\ 4 Department of Physics, University of Wisconsin, Madison, WI 53706, USA \\ 5 Gesellschaft für Schwerionenforschung mbH, D-64291 Darmstadt, Germany \\ 6 Laboratori Nazionali del Sud and INFN, I-95123, Catania, Italy \\ 7 NIPNE, RO-76900 Bucharest-Magurele, Romania \\ 8 Instituto de Fisica, Universidade Federal do Rio de Janeiro, Cidade Universitária, CP 68528, 21945-970 Rio de Janeiro, Brazil \\ 9 Cyclotron Institute, Texas A\&M University, College Station, TX 77843, USA
}

Original article: Eur. Phys. J. A 30, 129-139 (2006) DOI: 10.1140/epja/i2006-10111-0

Received: 22 May 2007

Published online: 29 May 2007 - (C) Società Italiana di Fisica / Springer-Verlag 2007

The two versions of MMM have been defined in reverse order.

Thus, on p. 130, second column, last paragraph, and p. 131, first column, first paragraph, the correct form is: "1) free-volume approach and 2) non-overlapping spherical fragments inside a spherical container."

Similarly, on p. 132, second column, first paragraph, the correct form is: "MMM1 which uses a free-volume parameterization emits nearly two fragments less than MMM2."

\footnotetext{
a e-mail: araduta@nipne.ro
} 